\title{
Scaling behavior of the self diffusion coefficients: dependence on the mass ratio of a binary mixture
}

\author{
Neeta Bidhoodi, and Shankar P. Das \\ School of Physical Sciences, \\ Jawaharlal Nehru University, \\ New Delhi 110067, India.
}

\begin{abstract}
We calculate the self diffusion coefficients $D_{S}$ for the species $s=1,2$ of a mixture, and show that a general scaling relation $D_{2} \sim D_{1}^{a}$ with a non universal exponent $a$ holds. The generalized diffusion coefficients, dependent on the mass ratio $\kappa=m_{2} / m_{1}$ of the constituent particles of the mixture, are computed using a proper formulation of the self-consistent mode coupling theory(MCT). The present model for the dense mixture includes nonlocal and nonlinear effects within the adiabatic approximation of fast decay of momentum fluctuations compared to the density fluctuations. The dependence of the slow dynamics near the characteristic ergodicity-nonergodicity (ENE) transition of the MCT on $\kappa$ is also studied and a reentrant behavior of of $D_{1}$ with respect to $D_{2}$, in agreement with simulations is obtained.
\end{abstract}


The time evolution in the fluctuation of a tagged particle density $\varrho(\mathbf{x}, t)$ in a fluid is a hydrodynamic process signifying the conservation of the particle. The decay of the time correlation $\psi(q, t)$ of the local density $\varrho(\mathbf{x}, t)$ is set by the corresponding self diffusion coefficient $D[1,3]$. The latter has been estimated through extensive experimental and numerical studies [2]. In a supercooled liquid a sharp fall in $D$ occurs signifying very slow single particle dynamics. Binary mixtures are often chosen in studying glassy dynamics and the behavior of the two self-diffusion coefficients $D_{s}$ corresponding to the respective species $s=1,2$ is an issue of much interest. Both $D_{1}$ and $D_{2}$ characterize the mixing process in the fluid and computer simulations[4] show that their behavior are strongly dependent of the mass ratio of the constituent particles of the mixture. Understanding this dependence of the slow dynamics involving $D_{1}$ and $D_{2}$ on the characteristic properties of the mixture, like size or mass asymmetry of its particles is the motivation of the present work.

A microscopic model for the correlated dynamics of the particles of a dense binary mixture has been formulated [5, 6] using the self-consistent mode coupling approach [7]. The mode coupling theory (MCT) in its simplest form[8, 10, 11] predicts a ENE transition in the mixture beyond a critical packing and has been used extensively in studying slow dynamics [9]. The model which takes in to account the proper conservation laws for the two component system shows that the transition point is dependent on the mass ratio [12] of the constituent particles. This dependence of the dynamics on the mass ratio has been seen in simulations [4]. By applying the so called adiabatic approximation in the MCT model, we presented [13] the mechanism for producing very slow dynamics of a tagged particle in an one component liquid. In the present work we show how the asymmetric mass ratio influences the selfdiffusion coefficients $D_{1}$ and $D_{2}$ of the two species of the mixture. The findings of our model are in agreement with simulation results.

The MCT is formulated primarily in terms of correlation of a small set of collective densities. Let us first consider the mechanism for producing very long relaxation time for the tagged particle correlation $\psi(q, t)$ in a one component fluid due to mode coupling effects. The MCT is formulated in this case in terms of the Laplace transform of the tagged particle correlation $\psi(q, t)$,

$$
\psi(q, z)=\left[z+i q^{2} D(q, z)\right]^{-1}
$$

where the generalized memory function $D(q, z)$ is written in the inverse form [13, 14] as $D^{-1}(q, z)=D_{0}^{-1}+\Delta_{m c}(q, z)$. Here $D_{0}$ is the bare self-diffusion coefficient and $\Delta_{m c}(q, z)$ is 
the mode coupling contribution. At the one loop order, the mode coupling part is obtained using the adiabatic approximation as

$$
\Delta_{m c}(q, z)=n_{0} \int \frac{d \mathbf{k}}{(2 \pi)^{3}} \frac{u^{2}}{k^{2}} V^{2}(q, k) \mathcal{C}(k) \psi(q-k, t),
$$

where $u$ is the cosine of the angle between $\mathbf{q}, \mathbf{k}$. The quantity $\mathcal{C}(k, t)$ is related to the collective density correlation function $\phi(q, t)$ as $\mathcal{C}(k, t)=\{c(k) \phi(k, t) c(k)\}$, where the weight factor $c(k)$ is Ornstein-Zernike two point direct correlation function normalized with a factor of average number density $n_{0}$. The vertex function $V(q, k)$ in the mode coupling integral of Eqn. (2) is obtained as, $V(q, k)=k_{B} T /\left(D_{0}(q) L_{0}(k)\right)$, where $L_{0}$ and $D_{0}$ respectively denote the corresponding bare viscosity and the bare self diffusion coefficient. Taking the transport coefficients for their respective hydrodynamic values, the vertex factor is like a characteristic length scale similar to that defined with respect to Stokes-Einstein relation [15]. By transforming Eqns. (11) and (2) to the time domain we obtain a coupled set of nonlinear integro-differential equations for $\psi(q, t)$ for different values of wave vector $q$. These equations contain time convolutions with the memory function $\Delta_{\mathrm{mc}}$. The latter is expressed in the mode coupling formulation in terms $\mathcal{C}$ which involves the correlation function $\phi(k, t)$ for the collective density. Thus to evaluate tagged particle correlation $\psi(q, z)$ and hence the self diffusion coefficient $D(q, z)$, we need to obtain independently the total correlation $\phi(q, z)$.

For the two component system, the relevant densities are the partial (mass) densities $\left\{\rho_{1}, \rho_{2}\right\}$ of the two species and the total momentum density $\mathbf{g}$. They respectively correspond to the conservation of mass of individual species and total momentum in the mixture. Linear combinations of $\rho_{1}$ and $\rho_{2}$ constitute an equivalent set of collective modes $\{\rho, c, \mathbf{g}\}$, where $\rho=\rho_{1}+\rho_{2}$ and $c=x_{1} \rho_{2}-x_{1} \rho_{1} . x_{s}$ is the fraction particles of species $s$. The theoretical analysis presented below, broadly consists of two parts. In the first part, we present the equations for the time evolution of the tagged particle correlations $\psi_{s}(q, t)$ for the mixture in terms of the corresponding memory functions $\Delta_{\mathrm{mc}}^{s}$ respectively for $s=1,2$. We approximate the respective memory functions in a self-consistent form in terms of convolutions of $\psi_{s}$ and the correlations of collective correlation functions. The latter are denoted as $\left\{\phi_{\alpha \sigma}\right\}$, with $\alpha, \sigma \in\{\rho, c\}$. In constructing these memory functions $\Delta_{\mathrm{mc}}^{s}$, we follow the corresponding results of MCT for a one component system obtained using the adiabatic approximation. In the second part of our analysis, we focus on the calculation of the collective correlation 
functions $\left\{\phi_{\alpha \sigma}\right\}$, with $\alpha, \sigma \in\{\rho, c\}$ needed for evaluating the self consistent expressions for the memory functions obtained in the first part. The self diffusion coefficients $D_{s}$, its dependence on the characteristic properties like mass ratio, size ratio, etc. are then computed.

Generalizing the results for the one component fluid, the corresponding equation of motion for the tagged particle auto-correlations $\psi_{s}(q, t)$ of species $s$ (for $\left.s=1,2\right)$ is obtained.

$$
\dot{\psi}_{s}(q, t)+D_{0}^{s} q^{2} \psi_{s}(q, t)+D_{0}^{s} \int_{0}^{t} d s \Delta_{m c}^{s}(q, t-s) \dot{\psi}_{s}(q, s)=0
$$

where $D_{0}^{s}$ denotes the bare diffusion coefficient for species $s$ [16]. The memory function $\Delta_{m c}^{s}(q, t)$ corresponding to $s$-th species is written in the form:

$$
\Delta_{m c}^{s}(q, t)=n_{0} \int \frac{d \mathbf{k}}{(2 \pi)^{3}} \frac{u^{2}}{k^{2}} V_{s}^{2}(q, k) \mathcal{C}_{s}(q, t) \psi_{s}(q-k, t)
$$

The vertex function $V_{s}(q, k)$ in the above equation is a generalization of the corresponding quantity $V(q, k)$ defined in Eqn. (2), with the self-diffusion coefficient $D_{0}$ being replaced by the corresponding $D_{0}^{s}$. The collective part of the correlation, $\mathcal{C}_{s}(k, t)$ in Eqn. (4), is obtained by generalizing the $\mathcal{C}(k, t)$ in the right hand side of Eq. (2) for the one component case. The $s s$-th element of the corresponding $2 \times 2$ matrix is written as

$$
\mathcal{C}_{s}(q, t)=\left[\mathbf{c}^{T}(k) \star \Phi(q, t) \star \mathbf{c}(k)\right]_{s s}
$$

$\Phi$ and $\mathbf{c}$ are the respective generalizations for the two component case [17] of the normalized density correlation $\phi(k, t)$ and Ornstein-Zernike direct correlation functions $c(k)$ for the one component fluid. The superscript $T$ in $\mathbf{c}^{T}$ stands for transpose of the $2 \times 2$ matrix $\mathbf{c}$ in in terms of species index $s, s^{\prime}$ etc. Similarly the correlation function matrix $\Phi$ is defined as $\Phi_{s s^{\prime}}(k, t)=$ $\left\{\sqrt{S_{s s}(k) S_{s^{\prime} s^{\prime}}(k)} \phi_{s s^{\prime}}(k, t)\right\}$, where, $\phi_{s s^{\prime}}$ is the equilibrium correlation of fluctuations of the partial densities $\rho_{s}(k, t)$ and $\rho_{s^{\prime}}(-k, t): \phi_{s s^{\prime}}(k, t)=\left\langle\delta \rho_{s}(k, t) \delta \rho_{s^{\prime}}(-k, 0)\right\rangle / \sqrt{N_{s} N_{s^{\prime}}}$. The matrix $S_{s s}(k)$ is the equal time correlation function $\phi_{s s^{\prime}}(k, t=0)(s=1,2) . \mathcal{C}^{s}(q, t)$ is the key quantity through which the collective modes of the mixture couple to the single particle modes in the respective memory function $\Delta_{m c}^{s}$ (for $\left.s=1,2\right)$. In writing Eqn. (3) we make the plausible assumption that the dynamics of the single particle modes $\left\{\psi_{s}\right\}$ of the respective species $s=1,2$ do not influence each other, and hence the time evolution of $\psi_{1}$ and $\psi_{2}$ are not linked. Each of the single particle correlations couple only to the collective modes in the mixture. 
The coupled set of Eqns. (3)-(4) are solved self-consistently on a wave vector grid to obtain the self correlation functions $\psi_{s}(q, t)$ (for $\left.s=1,2\right)$. The memory functions $\Delta_{m c}^{s}$ $(s=1,2)$ in the respective equations for $\psi_{s}(s=1,2)$ depend on $\mathcal{C}_{s}$ and hence on collective correlation functions $\left\{\phi_{\alpha \sigma}\right\}$. The latter are obtained from MCT for the binary mixture [6, 13] which follows from the corresponding equations of fluctuating nonlinear hydrodynamics. The renormalized theory which takes in to account the effect of the nonlinearities is constructed with the standard Martin-Siggia-Rose field theoretic approach. Using the available fluctuation dissipation relations between the correlation and response functions, we obtain the equations for the time evolutions of $\phi_{\alpha \sigma}$ with $\alpha, \sigma \in\{\rho, c\}$. The dynamics is considered here in the so called adiabatic approximation in which the momentum fluctuations decays out much faster than the density fluctuations. These details are provided in the Appendix .

The single particle dynamics becomes slaved to collective correlation in the adiabatic approximation as is evident from the coupled set of Eqns.(3) and (4). The renormalized self-diffusion coefficient for species $s(s=1,2)$ is obtained as $D_{s}=D_{0}^{s} /\left(1+\tilde{\Delta}_{s}\right)$ where $\tilde{\Delta}_{s}$ is the static limit of the corresponding memory function $\Delta_{m c}^{s}$, i.e., we write $\tilde{\Delta}_{s}=D_{0}^{s} \int_{0}^{\infty} \Delta_{m c}^{s}(0, t) d t$. The memory function $\Delta_{m c}^{s}(0, t)$ is expressed in terms of correlation functions of collective modes $\{\rho, c\}$ when the adiabatic approximation [13] is applied in the overdamped limit. Hence beyond the ENE transition $\Delta_{m c}^{s}(0, t)$ is constant over long times and we have $\tilde{\Delta}_{s} \rightarrow \infty$. Hence $D_{s} \rightarrow 0$. Single particle diffusion constants $D_{1}$ and $D_{2}$ vanish only with application of the adiabatic approximation. The location of the characteristic ENE transition is obtained from the long time behavior of the correlation of collective variables in the fluid. Beyond the ENE transition point $\phi_{\alpha \sigma}(q, t \rightarrow \infty)=f_{\alpha \sigma}(q)$ are non zero and this causes $\psi_{s}(q, t \rightarrow \infty)=f_{s}(q)$ (for $\left.s=1,2\right)$ being nonzero. $\left\{f_{\alpha \sigma}(q)\right\}$ and $f_{s}$ termed as nonergodicity parameters.

In the following, we present results for the dynamics of the hard sphere mixture from two broad aspects. First, we study the self-consistent MCT predictions for single particle correlations $\psi_{s}(q, t)$, and hence the self diffusion coefficients $D_{s}$ for $s=1,2$, when the mixture is in the ergodic phase. We study the dependence of MCT predictions for $D_{1}$ and $D_{2}$ on the mass ratio $\kappa=m_{2} / m_{1}$, size ratio $\alpha=\sigma_{2} / \sigma_{1}$ of the particles of the two species of the mixture, and the relative abundance $x=N_{2} / N$ of species 2. Second, we consider the characteristic ENE transition point of MCT and study how the critical value of total 
packing fraction $\eta_{c}$ changes with $\kappa, \alpha$ and $x$. In Fig. 1 we display $D_{1}$ and $D_{2}$ on a $\log$-log plot for binary mixture with size ratio $\alpha=1.2$ three different values of the mass ratio. The straight line fits indicate the two self diffusion coefficients $D_{1}$ and $D_{2}$ are simply related as $D_{1}=\vartheta D_{2}^{a}$ with the $a$ and $\vartheta$ being non-universal. As $\kappa$ increases the slope $a$ converges to a value close to 1.12. The study is done for a fixed value of the total packing fraction $\eta=.500$ of the mixture. Thus the variation of the diffusion constants along each curve, corresponds to a range of $x$. For lines shown in Fig. 1 corresponding to $\kappa=5,10$, and 20 the respective range of $x$ values are [.75-.85], [.85-.90]. In the inset of Fig. 1 we show diffusion data from simulation of mixture of soft spheres [18] having size ratio $\alpha=1.0$, and mass ratio $\kappa=10$. The diffusion data shown are at fixed packing $\eta=.313$ and temperature $T^{*}=1.05$, with the relative abundance $x$ varying over the range $[.05-.2]$. The straight lines for different $\kappa$ values in Fig. 1 are shifted along the horizontal axis so as to make each pass through the common point of the filled circle representing the one component system $(\alpha=1, \kappa=1)$. This shifting accounts for the amplitude factor $\vartheta(\kappa)$ in the scaling relation. For the three curves corresponding to $\kappa=5,10$, and 20, shown in Fig. 1 we obtain $\vartheta=15.0,3.5$, and 2.8. For the simulation data in the inset of Fig. 1 the same scaling holds with $\vartheta=.87$. In Fig. 2 we show the results of the MCT model for the diffusion constants for different packing fractions $\eta$ at a fixed value of relative abundance $x$. Mass ratio and size ratio characterizing the mixture are kept same for each set of data. The corresponding range of $\eta$ is $\{.500-.520\}$. The diffusion coefficients follow the behavior $D_{2} \sim D_{1}^{a}$ with $a=.96$. In the inset of Fig. 2 we show similar behavior seen in simulations of two different kinds of interaction potentials, namely, binary Lennard-Jones and Square well interaction [19].

Binary mixtures are very useful systems for studying glassy dynamics in computers, since they do not readily crystallize. An important issue in this regard is ease of equilibration for the mixture and this is controlled by its relaxation time. The dependence of the relaxation time $\tau$ on the mass ratio $\kappa$ of the mixture is shown in Fig. 3. The relaxation time $\tau$ is estimated from the time dependence of the decay of total density correlation $\psi_{\rho \rho}(q, t)$ at the peak of the corresponding structure factor $q_{m} \sigma_{2}=7.0$. The parameters of the mixture are kept fixed at $x=.95 \alpha=10$ and total packing fraction $\eta=.500$. As in Fig. 1 and Fig. 2, we study behavior of $\tau$ with change of either one of $x$ or $\kappa$, while the other is kept fixed. Generally as the bigger sized particles get more massive, relaxation slows down. On the other hand for the mixture of same species, i.e., constant $\kappa$ and $\alpha$, relaxation slows down as 
the mixture becomes more asymmetric in the relative abundance of the two species.

Finally we study the role of the mass ratio $\kappa$ on the possible ENE transition in the mixture. The location of the transition point, in terms of the critical value of the total packing fraction $\eta=\eta_{c}$, vs. relative abundance $x_{2}$ is shown for three different $\kappa$ in Fig. 4 , The size ratio for all the curves are fixed at $\alpha=1$. For the $\kappa=1$ we obtain for all $x$, the one component result showing that $\eta_{c}=.516$ independent of $x$. For $x=1$ or $x=0$, we have an one component system and hence the $\eta_{c}$ for the hard sphere system is fixed at .516. With increasing $\kappa$ however the ENE transition can occur at much lower packing at intermediate values of $x$. This is shown Fig. 4, In Ref. [4] the reentrant nature of the ENE transition was observed from simulations of a two component mixture. The system studied in Ref. [4] is not a hard sphere system. However we also observe similar behavior for a hard core system from our model equations. In Fig. 5 we plot the pairs of partial packing fraction values $\left\{\eta_{1}^{c}, \eta_{2}^{c}\right\}$ corresponding to the ENE transition of the MCT. Mixtures with three different $\kappa$ values, keeping the size ratio fixed at $\alpha=1$, are considered. In each case as the relative abundance changes the ENE transition occurs for different $\left\{\eta_{1}^{c}, \eta_{2}^{c}\right\}$ pairs. The nature of the criticality curves show that for certain $\eta_{2}$ values ( depending on $\kappa$ ) reentry in to the liquid phase occurs as $\eta_{1}$ is increased.

The mass ratio dependence of the dynamics which was absent in earlier MCT models, is seeded in the basic formulation of the present model. In earlier MCT formulations, the mass densities $\left\{\rho_{1}, \rho_{2}\right\}$, and the momentum densities $\left\{\mathbf{g}_{1}, \mathbf{g}_{2}\right\}$ of each component of the mixture were treated as slow variables. With the four "slow" variables $\left\{\rho_{s}, \mathbf{g}_{s}\right\}$, for $s=1,2$ the key nonlinearities in the corresponding equations of FNH (shown in the Appendix ) are $\rho_{s} \nabla_{i}\left(\delta F_{U} / \delta \rho_{s}\right)$. The mass dependence of the drops out in the corresponding formulation of the MCT. In subsequent works [4, 12] instead of $\left\{\mathbf{g}_{1}, \mathbf{g}_{2}\right\}$, only the conserved total momentum density $\mathbf{g}=\mathbf{g}_{1}+\mathbf{g}_{2}$ was taken as a slow mode. The corresponding set of Langevin equation for the mixture which gives rise to the mode coupling terms has the relevant non linearity in the equation for $\mathbf{g}$ of the form $\rho \nabla_{i}\left(\delta F_{U} / \delta \rho\right), c \nabla_{i}\left(\delta F_{U} / \delta c\right)$. This term is dependent on the mass ratio of the two species and hence the latter affects the dynamics. NB acknowledges CSIR, India, and SPD acknowledges the JC Bose fellowship of DST, for financial support.

[1] J. Boon, and S. Yip, 1991, Molecular Hydrodynamics, Dover, New York. 
[2] W. Kob and H. C. Andersen, 1995, Phys. Rev. E 51, 4626; ibid. 52, 4134 (1995).

[3] S. P. Das, Statistical Physics of Liquids at Freezing and Beyond, Cambridge University Press, NewYork, (2011).

[4] E. Zaccarelli, H. Lőwen, P. P. F. Wessels, F. Sciortino, P. Tartaglia, and C. N. Likos, Phys. Rev. Lett. 92, 225703 (2004).

[5] U. Harbola and S. P. Das, Phys. Rev. E 65, 036138 (2002).

[6] M. Priya, N. Bidhoodi, and S. P. Das, Physical Review E 92, 062308 (2015).

[7] S.P. Das, Reviews of Modern Physics 76, 785-849 (2004).

[8] J. Bosse and J. S. Thakur, Phys. Rev. Lett. 59, 998 (1987).

[9] E. Rabani, K. Miyazaki, D. Reichman, J Chem Phys. 122 34502(2005).

[10] W. Götze and Th. Voigtmann, Phys. Rev. E 67, 021502 (2003).

[11] J-L Barrat, and A. Latz, J. Phys. Condens. Matter 2, 4289 (1990).

[12] U. Harbola, and S. P. Das, J. Stat. Phys. 112, 1131 (2003).

[13] N. Bidhoodi and S. P. Das Physical Review E 92, 062309 (2015).

[14] K. Kawasaki, Physica A 215, 61 (1995).

[15] G. Biroli and J-P Bouchaud, J. Phys. Cond. Matt. 19205101 (2007).

[16] S. Sinha and M. C. Marchetti, Phys. Rev. A 46, 4942 (1992).

[17] U. Krieger and J. Bosse, Phys. Rev. Lett. 59, 1601 (1987).

[18] W. Fenz, I. M. Mryglod, O. Prytula, and R. Folk, Phys. Rev. E 80, 021202 (2009).

[19] A. D. S. Parmar, S. Sengupta, and S. Sastry, Phys. Rev. Lett. 119, 056001 (2017).

[20] C. Cohen, J. W. H. Sutherland and J. M. Deutch, Phys. and Chem. of Liquids, 2, 213 (1971).

[21] A. Andreanov, G. Biroli, and A. Lefevre, J. Stat. Mech, P07008 (2006).

[22] S. P. Das, Physical Review E, 541715 (1996).

[23] S. P. Das and G. F. Mazenko, Phys. Rev. E. 79, 021504 (2009).

[24] S. P. Das, and J. W. Dufty, Physical Review A 46, 6371 (1992).

[25] J. K. Percus, and G. J. Yevick, Phys Rev 1, 1103 (1958).

[26] J. L. Lebowitz, Phys.Rev. 133, A895 (1964).

[27] S. P. Das and G. F. Mazenko, Journal of Statistical Physics 152, 159194 (2013). 


\section{Appendix}

The coupled dynamics of the time correlation functions $\phi_{\alpha \sigma}(q, t)$ of the collective modes $\alpha, \sigma \in\{\rho, c\}$ is controlled by the equations of fluctuating nonlinear hydrodynamics (FNH) for a mixture. These equations signify the basic conservation laws for the system and involve nonlinear couplings of the collective modes. We explain here the steps of calculating the correlation functions of the collective densities for a binary mixture of $N_{s}$ identical particles of species $s$ having mass $m_{s}$ for $s=1,2$ respectively. The concentration of the species $s$ is $x_{s}=N_{s} / N$ and $N=N_{1}+N_{2}$ is the total number of particles. The individual mass densities $\rho_{s}$ and the momentum densities $\mathbf{g}_{s}$ for the species $s$ are respectively defined in terms of microscopic phase space variables as follows,

$$
\begin{aligned}
& \rho_{s}(\mathbf{x}, t)=m_{s} \sum_{i=1}^{N_{s}} \delta\left(\mathbf{x}-\mathbf{R}_{s}^{i}(t)\right), \\
& \mathbf{g}_{s}(\mathbf{x}, t)=\sum_{i=1}^{N_{s}} \mathbf{p}_{s}^{i} \delta\left(\mathbf{x}-\mathbf{R}_{s}^{i}(t)\right) .
\end{aligned}
$$

The phase space coordinates of position and momentum of the $i$-th particle of the species $s$ are denoted as $\left\{R_{s}^{i}(t), P_{s}^{i}(t)\right\}$. The individual mass densities, respectively denoted as $\rho_{s}(\mathbf{x}, t)$ are microscopically conserved. The individual momentum densities $\mathbf{g}_{1}$ and $\mathbf{g}_{2}$ are not conserved but total momentum density defined as, $\mathbf{g}(\mathbf{x}, t)=\mathbf{g}_{1}(\mathbf{x}, t)+\mathbf{g}_{2}(\mathbf{x}, t)$ is conserved. In the following we consider the dynamics in terms of a different set of conserved variables: the total mass density $\rho(\mathbf{x}, t)$, total momentum density $\mathrm{g}(\mathbf{x}, t)$, and the concentration variable $c(\mathbf{x}, t)[20]$. The mass and concentration densities are defined as follows:

$$
\begin{aligned}
\rho(\mathbf{x}, t) & =\rho_{1}(\mathbf{x}, t)+\rho_{2}(\mathbf{x}, t), \\
c(\mathbf{x}, t) & =x_{2} \rho_{1}(\mathbf{x}, t)-x_{1} \rho_{2}(\mathbf{x}, t) .
\end{aligned}
$$

We define the fluctuations of $\rho$ and $c$ respectively as $\delta \rho=\rho-\rho_{0}$ and $\delta c=c$, since the average of $c$ is zero when we consider the mass ratio of the constituent particles to be unity.

The generalized Langevin equations for the respective coarse grained densities $\{\rho(\mathbf{x}, t), \mathbf{g}(\mathbf{x}, t), c(\mathbf{x}, t)\}$ for a binary mixture are obtained following standard procedures [3].

$$
\frac{\partial \rho}{\partial t}+\nabla \cdot \mathbf{g}=0
$$




$$
\begin{aligned}
& \frac{\partial \mathrm{g}_{i}}{\partial t}+\nabla_{j}\left[\frac{\mathrm{g}_{i} \mathrm{~g}_{j}}{\rho}\right]+\rho \nabla_{i} \frac{\delta F_{U}}{\delta \rho}+c \nabla_{i} \frac{\delta F_{U}}{\delta c}+L_{i j}^{0} \frac{\mathrm{g}_{j}}{\rho}=\theta_{i}, \\
& \frac{\partial c}{\partial t}+\nabla \cdot\left[c \frac{\mathbf{g}}{\rho}\right]+\gamma_{c c} \nabla^{2} \frac{\delta F_{U}}{\delta c}=f_{c} .
\end{aligned}
$$

$F_{U}$ is the so called potential part of the free energy functional $F$ which is expressed as

$$
F[\rho, \mathbf{g}, c]=F_{K}[\rho, \mathbf{g}]+F_{U}[\rho, c],
$$

where the kinetic part $F_{K}$ (dependent on the current density $\mathbf{g}$ ) is computed from the microscopic Hamiltonian for the $N$ particle mixture [5]. $F_{U}[\rho, c]$ is taken here as a quadratic functional of the fields $\rho$ and $c$, and is related to the structure of the liquid. This is expressed in terms of the corresponding direct correlation functions $\left\{c_{\rho \rho}, c_{\rho c}, c_{c c}\right\}$ defined in the Ornstein-Zernike relations. $L_{i j}^{0}$ represents the matrix of bare or short time viscosities while $\gamma_{c c}$ links to the bare inter-diffusion coefficient for the mixture. These two dissipative coefficients are related to the correlation of the Gaussian noises respectively in Eqs. (6) and (7) through standard fluctuation-dissipation relations [6].

Effects of these nonlinearities on the correlation functions are obtained using a MartinSiggia-Rose (MSR) field theory. In this formulation the renormalized transport coefficients are obtained in a self consistent form[6, 13]. Applying the available fluctuation dissipation relations [21] in the model and introducing the the intermediate kernel function $\Xi(q, t)$, the equations of motion for correlation functions $\phi_{\alpha \sigma}$ are obtained as follows:

$$
\begin{aligned}
& \dot{\phi}_{\rho \rho}(q, t)+\Omega_{q}^{2} \int_{0}^{t} d s \Xi(q, t-s) \tilde{\phi}_{\rho \rho}(q, s)=0, \\
& \dot{\phi}_{\rho c}(q, t)+\Omega_{q}^{2} \int_{0}^{t} d s \Xi(q, t-s) \tilde{\phi}_{\rho c}(q, s)=0, \\
& \dot{\Xi}(q, t)+\Omega_{q}^{2}\left\{L_{0}(q) \Xi(q, t)+\int_{0}^{t} d s L(q, t-s) \Xi(q, s)\right\}=0, \\
& \dot{\phi}_{c c}(q, t)+\nu_{0} q^{2} \tilde{\phi}_{c c}(q, z)+\int_{0}^{t} d s M(q, t-s) \dot{\phi}_{c c}(q, s)=0 .
\end{aligned}
$$

The quantity $\Omega_{q}=q c_{0}$ represents a characteristic frequency scale for the mixture in terms of the sound speed $c_{0}$ and we have defined the dressed correlations: $\tilde{\phi}_{\rho \alpha}=\phi_{\rho \alpha}-\chi \phi_{c \alpha}$ and $\tilde{\phi}_{c c}=$ $\phi_{c c}-\chi \phi_{\rho c}$, with $\chi=\chi_{\rho c} / \sqrt{\chi_{\rho \rho} \chi_{c c}}, \alpha \in\{\rho, c\} . L_{0}(q)$ is the bare longitudinal viscosity to which a mode coupling contribution $L(q, t)$ is added. Similarly the inverse of the inter-diffusion coefficient of the mixture includes a mode coupling contribution $M(q, t)$. In writing Eqns. (99)-(12), the presence of $1 / \rho$ nonlinearities [22, 23] in the FNH equations for a mixture have 
been ignored. The memory functions $M(q, t)$ and $L(q, t)$ - mode-coupling expressions in terms of correlations involving $\{\rho, c\}$. In the adiabatic approximation relevant for the glassy dynamics, the simplest mode coupling expressions for $L(q, t)$ and $M(q, t)$ are obtained as bilinear products involving correlation functions $\phi_{\alpha \sigma}$ of the fields $\{\rho, c\}$. These coupled set of integro-differential equations are solved with the initial conditions $\psi_{\rho \rho}(q, 0)=1$, $\psi_{\rho c}(q, 0)=\chi$ and $\psi_{c c}(q, 0)=1$. The coupled dynamics of the correlations of the $\{\rho, c\}$ fields is strongly dependent on the mass ratio $\kappa$ of the mixture since the effective nonlinearities and hence the memory functions in Eqns. (91)-(11) involves $\kappa$ in the present model. Beyond a ENE transition point $\phi_{\alpha \sigma}(q, t \rightarrow \infty)=f_{\alpha \sigma}(q)$ are non zero. $\left\{f_{\alpha \sigma}(q)\right\}$ are termed as nonergodicity parameters for collective modes. In solving the Eqns. (9)-(11), the bare transport coefficients $L_{0}(q)$ and $\gamma_{0}(q)$ are estimated in terms of those of a hard sphere system, obtained from short time models [24] of generalized hydrodynamics. Here the Percus-Yevick [25] structure factors for the mixture [26] is used. The present model predicts an ergodicitynonergodicity transition at a critical packing [27]. In the non-ergodic state the long time limits of the correlations of $\{\rho, c\}$ fields are nonzero.

Next we turn to the tagged particle correlations $\psi_{s}(q, t)$ for the two species $s=1,2$. The corresponding time evolution equations ( described in the main text) involve convolutions with the respective memory functions $\Delta_{\mathrm{mc}}^{s}(q, t)$ for $s=1,2$. These memory functions for the tagged particle correlations has mode coupling contributions similar to that in the memory functions for the one component fluid obtained in the adiabatic approximation [13]. For both one component or multi component systems, the mode coupling effect slaves the single particle dynamics to the collective dynamics in the dense system. Since the mode-coupling dynamics of collective variables are dependent on the mass ratio [6, 12, 13] of the mixture, similar dependence occurs for tagged particle correlations $\psi_{s}(q, t)(s=1,2)$ and hence for the self diffusion coefficients $D_{s}(s=1,2)$. The collective correlation contributions $\mathcal{C}_{s}, s=1,2$ present in the respective memory functions, are obtained by solving Eqns. (9)-(11) outlined above. To compute $\mathcal{C}_{s}$ in terms of the collective correlation functions, we work in terms of the conserved densities $\alpha, \sigma \in\{\rho, c\}$ rather than the partial densities $\rho_{s}$ of species $s=1,2$. The collective quantities $\mathcal{C}_{s}(s=1,2)$ are obtained in terms of the $\phi_{\alpha \sigma}$ as

$$
\mathcal{C}_{s}(q, t)=\sum_{\alpha \sigma} \tilde{c}_{\rho \alpha}^{(s)}(q) \tilde{c}_{\rho \sigma}^{(s)}(q) \sqrt{S_{\alpha \alpha}(q) S_{\sigma \sigma}(q)} \phi_{\alpha \sigma}(q, t)
$$

The functions $\tilde{c}_{\alpha \sigma}^{(s)}$ ( for $\left.\alpha, \sigma \in\{\rho, c\}\right)$ are defined as $\tilde{c}_{\rho \alpha}^{(s)}(q)=c_{\rho \alpha}(q)+(-1)^{s}\left(x_{s}-1\right) c_{\alpha c}(q)$, for 
$s=1,2$. The normalized time correlations $\phi_{s s^{\prime}}$, and direct correlations $c_{s s^{\prime}}\left(\right.$ for $\left.s, s^{\prime}=1,2\right)$ are easily transformed to their counterparts $\phi_{\alpha \sigma}$ and $c_{\alpha \sigma}$, where $\alpha, \sigma \in\{\rho, c\}$. Beyond a ENE transition point $\psi_{s}(q, t \rightarrow \infty)=f_{s}(q)$ (for $\left.s=1,2\right)$ and $f_{s}$ termed as nonergodicity parameters. The self diffusion constant goes to zero beyond the ENE transition.

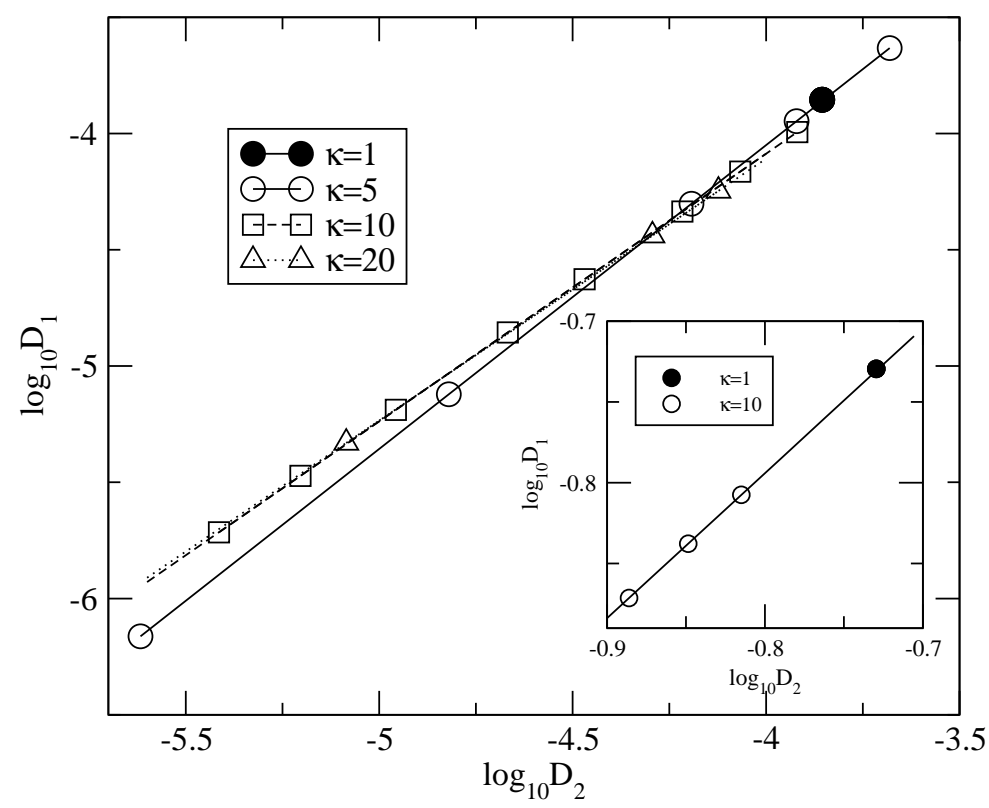

FIG. 1: Self-diffusion coefficients (expressed in units of $\sigma_{2} / \sqrt{\beta m_{1}}$ ) obtained from MCT for a hard sphere mixture with size ratio $\alpha=1$ and respective mass ratios $\kappa=$ 5(circle),10(square),20(triangle). The plots follow the scaling relation $D_{2}=\vartheta D_{1}^{a}$ with $a=1.30$, 1.15, and 1.12 respectively. Inset shows simulation results of a truncated and shifted Lennard-Jones mixture for $\alpha=1$ and $\kappa=10$ (reproduced from Ref. [18]). The straight lines for different $\kappa$ values are shifted along the horizontal axis so as to pass through the common point of the filled circle representing the one component system. The shifting is accounted for by the respective amplitude factors $\vartheta(\kappa)$ in the scaling relation. 


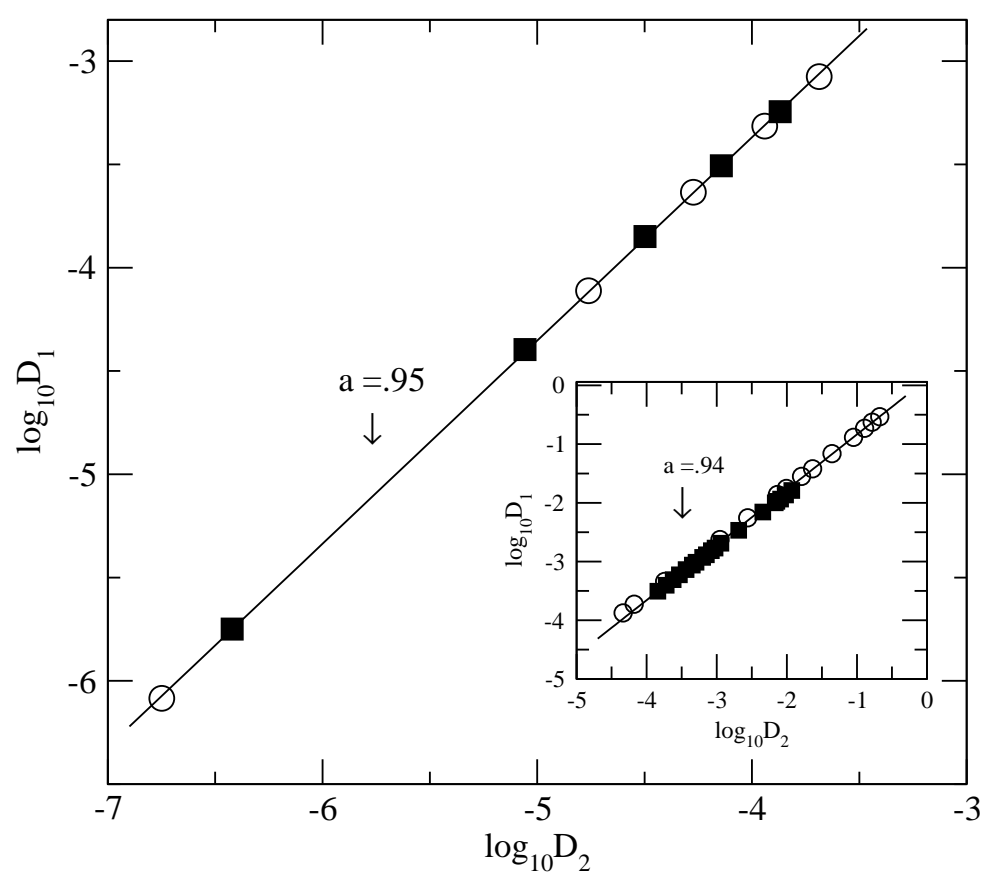

FIG. 2: Self-diffusion coefficients (expressed in units of $\sigma_{2} / \sqrt{\beta m_{1}}$ ) obtained from MCT for a binary mixture of hard spheres of size ratio $\alpha=1.2$, mass ratio $\kappa=1$ and relative abundance $x=$ .8 (circle),.5(square). The data corresponds to the total packing fraction $\eta$ range $\{.500-.520\}$. Inset shows simulation results (reproduced from Ref. [19]) with circle(square) for a Kob-Anderson(Square Well) mixture with size ratio $\alpha=1.25(1.20)$, and mass ratio $\kappa=1(1)$. The range of temperatures, scaled with respect to the corresponding potential depth $\epsilon$, are $\{0.46,6.0\}(\{0.31-10\})$. The scaling exponents are $a=.95$ (main) and .94 (inset). 


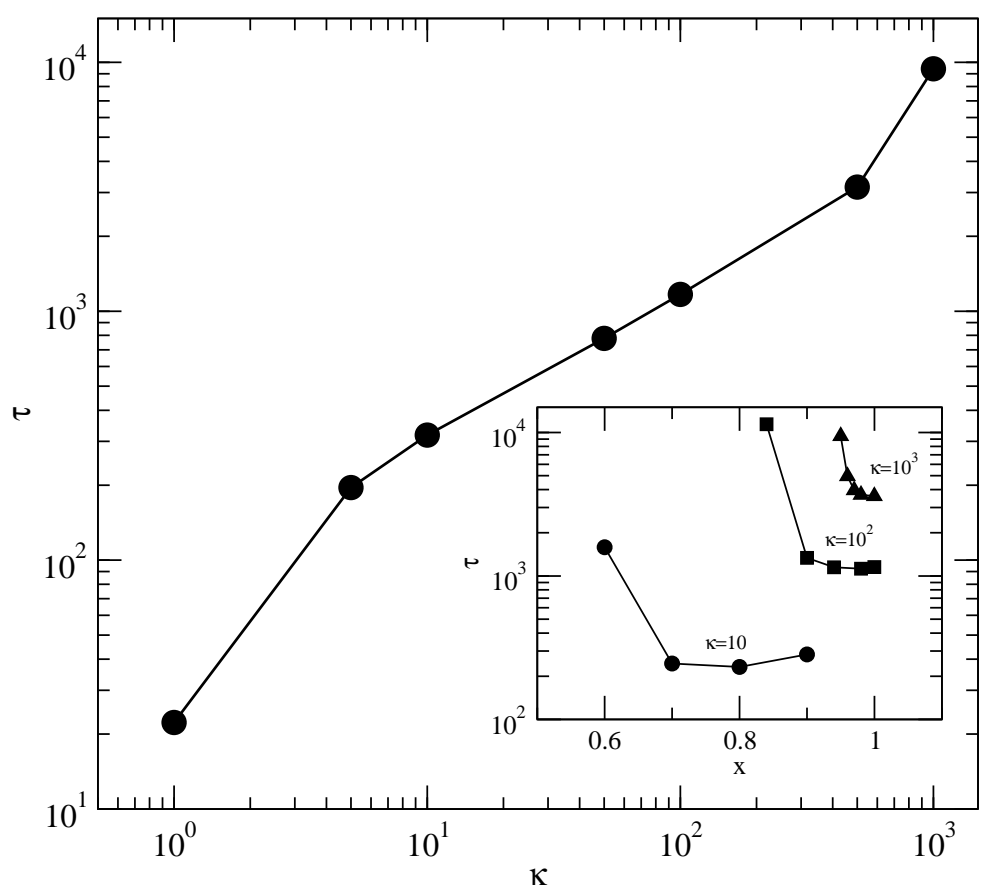

FIG. 3: Relaxation time $\tau$ for the total density correlation $\psi_{\rho \rho}$ near the structure factor peak vs. mass ratio $\kappa$ for a mixture of hard spheres, with size ratio $\alpha=10$, relative abundance $x=.95$ and total packing fraction .5. Inset: $\tau$ vs. $x$ for size ratio $\alpha=10$. The curves are labeld with corresponding mass ratio $\kappa$ 


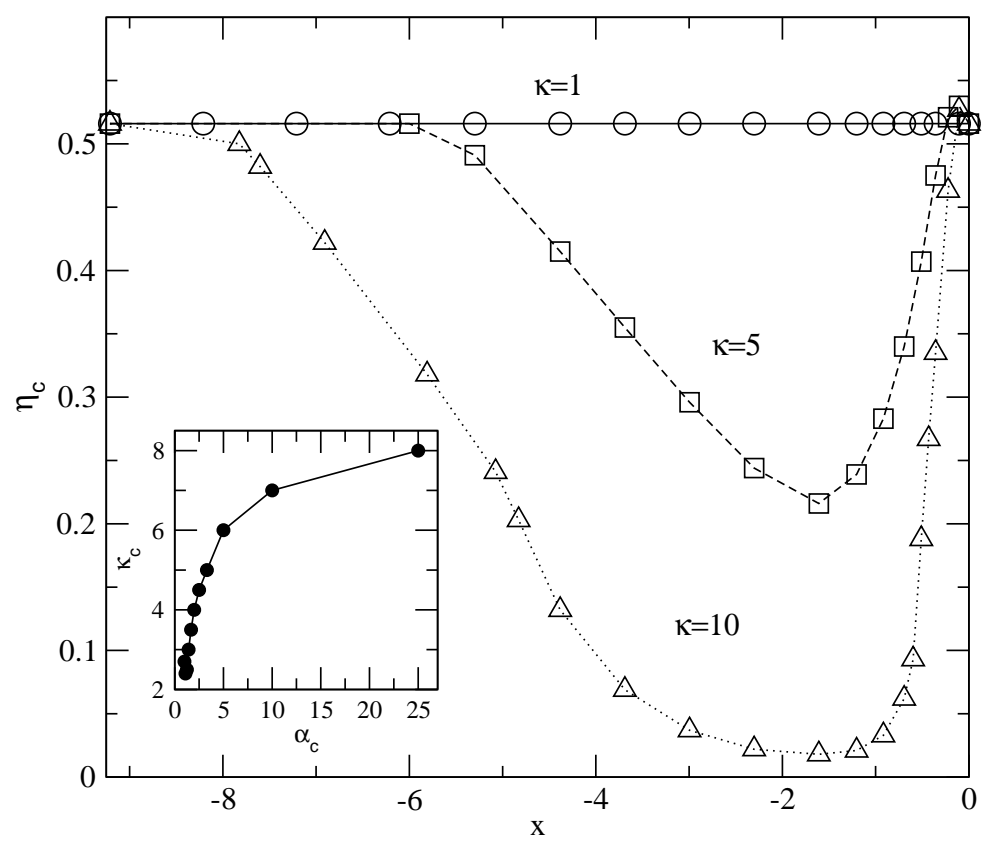

FIG. 4: The critical packing fraction $\eta_{c}$ corresponding to the ENE transition of MCT vs. the relative abundance $x$ of a hard sphere mixture having size ratio $\alpha=1$, and mass ratio $\kappa=1$ (circle), 5 (squares), and 10 (traingles). Inset shows for a mixture with packing $\eta=.5$ and $x=.5$, the pairs of values for mass ratio $\kappa_{c}$ and corresponding size ratio $\alpha_{c}$ at the ENE transition of MCT. 


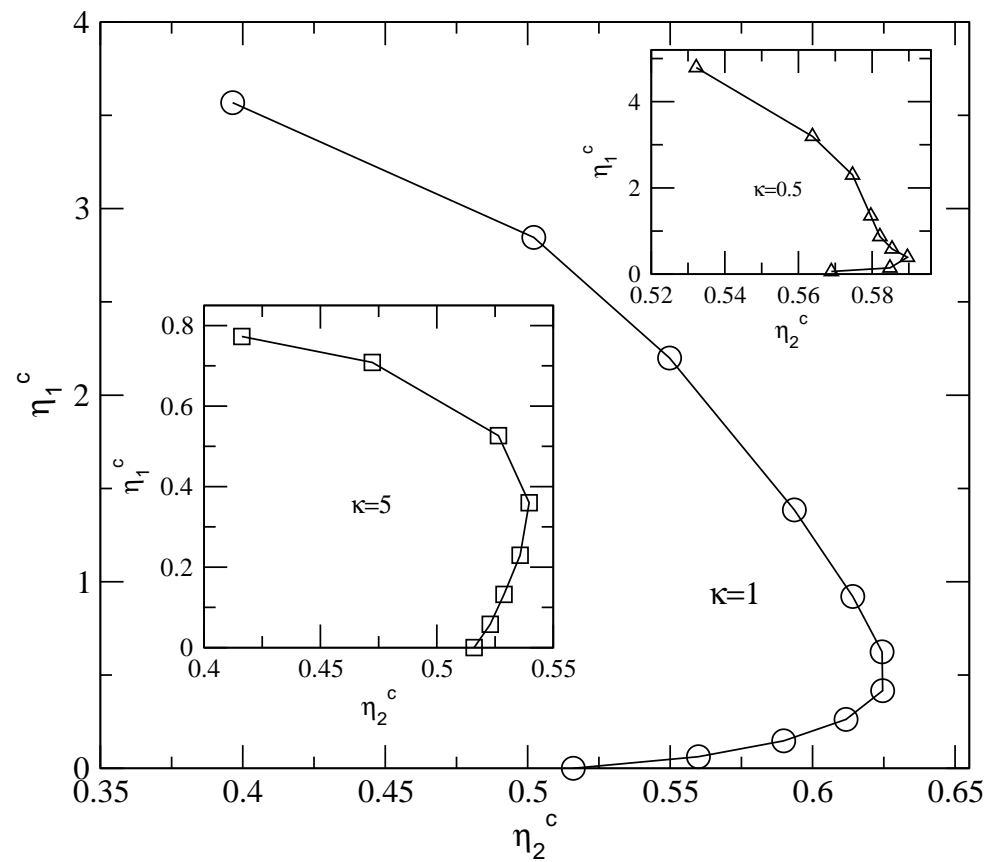

FIG. 5: The respective packing fractions of the two species at the ENE transition points of MCT, $\left\{\eta_{1}^{c}, \eta_{2}^{c}\right\}$ for a hard sphere mixture of size ratio $\alpha=10$ and mass ratio $\kappa=1$ (main), .5 (upper inset), and 5 (lower inset). Reentry of the critical behavior with respect to $\eta_{2}^{c}$ is seen here since at a fixed $\eta_{2}^{c}$, two possible values of $\eta_{1}^{c}$ occur. 